\title{
Some revisited results about composition operators on Hardy spaces
}

\author{
Pascal Lefèvre, Daniel Li, Hervé Queffélec, Luis Rodríguez-Piazza
}

\begin{abstract}
On the one hand, we generalize some results known for composition operators on Hardy spaces to the case of Hardy-Orlicz spaces $H^{\Psi}$ : construction of a "slow" Blaschke product giving a non-compact composition operator on $H^{\Psi}$ and yet "nowhere differentiable"; construction of a surjective symbol whose associated composition operator is compact on $H^{\Psi}$ and is, moreover, in all Schatten classes $S_{p}\left(H^{2}\right), p>0$. On the other hand, we revisit the classical case of composition operators on $H^{2}$, giving first a new, and simpler, characterization of composition operators with closed range, and then showing directly the equivalence of the two characterizations of membership in Schatten classes of Luecking, and Luecking-Zhu.
\end{abstract}

\section{Introduction}

The study of composition operators on Hardy spaces is now a classical subject (see [3] and [18] for example). In [8] (see also [7]), we considered a more general setting and studied composition operators on Hardy-Orlicz spaces; we gave there a characterization of their compactness in terms of the Carleson function of their symbol (and in terms of the Nevanlinna counting function in [11]). This work was continued in [10]: we compared compactness on Hardy spaces with compactness on Hardy-Orlicz spaces. For instance, we showed that for every $1 \leq p<\infty$, there is an Orlicz function $\Psi$ such that $H^{p+\varepsilon} \subseteq H^{\Psi} \subseteq H^{p}$ for every $\varepsilon>0$, and a composition operator $C_{\varphi}$ such that $C_{\varphi}$ is compact on $H^{p}$ and $H^{p+\varepsilon}$, but not compact on $H^{\Psi}$.

We carry on this study in the present work, taking advantage of our previous results ([8] and [11]). In a first part (Section 3 and Section 4), we shall improve, and extend to the Hardy-Orlicz case, results known for Hardy spaces; we use there results of [8]. In a second part (Section 5 and Section 6), we shall give new lights on some results concerning Hardy spaces, using the results of [11] establishing the

Mathematics Subject Classification (2010): Primary 47B33; Secondary 30H10, 30J10, 47B10.

Keywords: Blaschke product, Carleson function, Carleson measure, composition operator, Hardy-Orlicz space, Nevanlinna counting function, Schatten classes. 
equivalence of the Nevanlinna counting function with the Carleson function of the symbol. More precisely, the content of this paper is as follows.

B. MacCluer ${ }^{1}$ and J. Shapiro (Theorem 3.10 of [14]; see also $\S 3.2$ of [18]) proved that, when their symbol $\varphi$ is finitely-valent, the compactness of composition operators $C_{\varphi}$ on the Hardy space $H^{2}$ can be characterized by the behaviour of the modulus of $\varphi$ near the frontier of $\mathbb{D}$ : compactness is equivalent to the condition $1-|z|=o(1-|\varphi(z)|)$ as $|z| \rightarrow 1$. But the latter does not imply compactness in general (Example 3.8 of [14]; see also $§ 10.2$ of [18]). In Theorem 5.3 of [11], we gave such a characterization for composition operators, with finitely-valent symbol, on Hardy-Orlicz spaces. In Section 3, we construct a "slow" Blaschke product (generalizing $§ 10.2$ of [18] and Proposition 5.5 of [8]) showing that this condition is not sufficient in general. In Section 4, we construct a compact composition operator $C_{\varphi}: H^{\Psi} \rightarrow H^{\Psi}$ with surjective symbol $\varphi$ and such that $C_{\varphi}: H^{2} \rightarrow H^{2}$ is in all Schatten classes $S_{p}\left(H^{2}\right), p>0$. This generalizes and improves a result of B. MacCluer and J. Shapiro (Example 3.12 in [14]; see also $§ 2$ in the survey [16]). In Section 5, we give a characterization of composition operators $C_{\varphi}: H^{p} \rightarrow H^{p}$, $1 \leq p<\infty$, with a closed range, simpler than the former ones (see [1] and [20]). Finally, based on the main result of [11], we show directly, in Section 6, the equivalence of Luecking's and Luecking-Zhu's criteria ([12], [13]) for the membership of $C_{\varphi}: H^{2} \rightarrow H^{2}$ in Schatten classes.

\section{Notation}

The open unit disk is denoted by $\mathbb{D}=\{z \in \mathbb{C} ;|z|<1\}$ and its boundary, the unit circle, by $\mathbb{T}=\{z \in \mathbb{C} ;|z|=1\}$. The normalized Lebesgue measure $d t / 2 \pi$ on $\mathbb{T}$ is denoted by $m$. The normalized area measure $d x d y / \pi$ is denoted by $A$.

The Hardy space $H^{1}$ is the space of analytic functions $f: \mathbb{D} \rightarrow \mathbb{C}$ such that $\sup _{r<1} \int_{0}^{2 \pi}\left|f\left(r \mathrm{e}^{i \theta}\right)\right| d \theta<\infty$. Every $f \in H^{1}$ has almost everywhere non-tangential limits on $\mathbb{T}$, and we denote by $f^{*}$ the function on $\mathbb{T}$ given by these boundary values.

An Orlicz function is a convex nondecreasing function $\Psi:[0, \infty) \rightarrow[0, \infty)$ such that $\Psi(0)=0$ and $\Psi(\infty)=\infty$. If $\mu$ is a positive measure on some measurable space $S$, the Orlicz space $L^{\Psi}(\mu)$ is the set of all (classes of) measurable functions $f: S \rightarrow \mathbb{C}$ such that $\int_{S} \Psi(|f| / C) d \mu<\infty$ for some $C>0$; the norm $\|f\|_{\Psi}$ is defined as the infimum of all positive numbers $C$ for which $\int_{S} \Psi(|f| / C) d \mu \leq 1$.

The Hardy-Orlicz space $H^{\Psi}$ is the linear subspace of $f \in H^{1}$ such that $f^{*} \in$ $L^{\Psi}(m)$ (see [8]).

Every analytic self-map $\varphi: \mathbb{D} \rightarrow \mathbb{D}$ defines a bounded composition operator $C_{\varphi}: f \in H^{\Psi} \mapsto f \circ \varphi \in H^{\Psi}$ (see [8]).

For every $\xi \in \mathbb{T}$ and $0<h<1$, we set

$$
S(\xi, h)=\{z \in \mathbb{D} ;|z-\xi| \leq h\} .
$$

\footnotetext{
${ }^{1}$ We misspelled her name in our previous papers, and we apologize for this.
} 
The Carleson function $\rho_{\varphi}$ of the analytic self-map $\varphi: \mathbb{D} \rightarrow \mathbb{D}$ is defined, for $0<h<1$, by

$$
\rho_{\varphi}(h)=\sup _{\xi \in \mathbb{T}} m\left(\left\{\mathrm{e}^{i \theta} \in \mathbb{T} ; \varphi^{*}\left(\mathrm{e}^{i \theta}\right) \in S(\xi, h)\right\}\right)
$$

Alternatively, $\rho_{\varphi}(h)=\sup _{\xi \in \mathbb{T}} m_{\varphi}[S(\xi, h)]$, where $m_{\varphi}$ is the pull-back measure of $m$ by $\varphi$. We shall sometimes use the classical Carleson window, namely the set $W(\xi, h)=\{z \in \mathbb{D} ;|z| \geq 1-h$ and $|\arg (z \bar{\xi})| \leq h\}$ instead of $S(\xi, h)$, because it has an equivalent size.

The Nevanlinna counting function $N_{\varphi}$ is defined, for $w \in \varphi(\mathbb{D}) \backslash\{\varphi(0)\}$, by

$$
N_{\varphi}(w)=\sum_{\varphi(z)=w} \log \frac{1}{|z|},
$$

each term $\log \frac{1}{|z|}$ being repeated according to the multiplicity of $z$. It may be extended to the whole of $\mathbb{D}$ by $N_{\varphi}[\varphi(0)]=+\infty$ and $N_{\varphi}(w)=0$ for $w \in \mathbb{D} \backslash \varphi(\mathbb{D})$.

\section{Slow Blaschke products}

B. MacCluer and J. Shapiro (Theorem 3.10 of [14]; see also §3.2 in [18]) proved that, when $\varphi$ is finitely-valent (meaning that, for some $s \geq 1$, the equation $\varphi(z)=w$ has at most $s$ solutions), the composition operator $C_{\varphi}: H^{p} \rightarrow H^{p}$ is compact, $1 \leq p<\infty$, if and only if $\varphi$ has an angular derivative at no point of $\mathbb{T}$; that means that

$$
\lim _{|z| \rightarrow 1} \frac{1-|z|}{1-|\varphi(z)|}=0 .
$$

In Theorem 5.3 of [11], we generalized this result to Hardy-Orlicz spaces and proved that if $\varphi$ is finitely-valent, the composition operator $C_{\varphi}: H^{\Psi} \rightarrow H^{\Psi}$ is compact if and only if

$$
\lim _{|z| \rightarrow 1} \frac{\Psi^{-1}\left[\frac{1}{1-|\varphi(z)|}\right]}{\Psi^{-1}\left[\frac{1}{1-|z|}\right]}=0
$$

Without the assumption that $\varphi$ is finitely-valent, condition (3.2) is no longer sufficient to ensure the compactness of $C_{\varphi}: H^{\Psi} \rightarrow H^{\Psi}$. Indeed, we are going to construct a Blaschke product satisfying (3.2), but whose associated composition operator is of course not compact on $H^{\Psi}$, as this is the case for every inner function. A Blaschke product satisfying (3.1) is constructed in $\S 10.2$ of [18]; that construction uses Frostman's Theorem. Our construction, which is more general, is entirely elementary. 
Theorem 3.1. Let $\delta:(0,1) \rightarrow(0,1 / 2]$ be any function such that $\lim _{t \rightarrow 0} \delta(t)=0$. Then, there exists a Blaschke product $B$ such that

$$
1-|B(z)| \geq \delta(1-|z|), \quad \text { for all } z \in \mathbb{D} .
$$

Corollary 3.2. For every Orlicz function $\Psi$ there exists a Blaschke product $B$ which satisfies

$$
\lim _{|z| \rightarrow 1} \frac{\Psi^{-1}\left[\frac{1}{1-|B(z)|}\right]}{\Psi^{-1}\left[\frac{1}{1-|z|}\right]}=0
$$

though the composition operator $C_{B}: H^{\Psi} \rightarrow H^{\Psi}$ is not compact.

Proof. $C_{B}$ is not compact since every compact composition operator should satisfy $\left|\varphi^{*}\right|<1$ a.e. (see Lemma 4.8 of [8]). It suffices then to choose $\delta(t)=$ $1 / \Psi\left(\sqrt{\Psi^{-1}(1 / t)}\right)$, which satisfies the hypothesis of Theorem 3.1. Moreover,

$$
\frac{\Psi^{-1}(1 / \delta(t))}{\Psi^{-1}(1 / t)}=\frac{1}{\sqrt{\Psi^{-1}(1 / t)}} \underset{t \rightarrow 0}{\longrightarrow} 0,
$$

and condition (3.3) gives the result.

Proof of Theorem 3.1. We shall essentially construct our Blaschke product $B$ as an infinite product of finite Blaschke products

$$
\prod_{n} B_{n}
$$

where each finite Blaschke product $B_{n}$ has $p_{n}$ zeros equidistributed in the circumference of radius $r_{n}$. That is, we will have, writing $\theta_{k}=2 \pi k / p_{n}$ and $z_{k}=r_{n} \mathrm{e}^{i \theta_{k}}$, for $k=1,2, \ldots, p_{n}$,

$$
B_{n}(z)=\prod_{k=1}^{p_{n}} \frac{\left|z_{k}\right|}{z_{k}} \frac{z_{k}-z}{1-\bar{z}_{k} z}=\prod_{k=1}^{p_{n}} \frac{r_{n}-\mathrm{e}^{-i \theta_{k}} z}{1-r_{n} \mathrm{e}^{-i \theta_{k} z}} .
$$

We shall need the following estimate for the finite Blaschke product in (3.4).

Lemma 3.3. Let $p \in \mathbb{N}$, and $0<r<1$. Consider the finite Blaschke product

$$
G(z)=\prod_{k=1}^{p} \frac{r-\mathrm{e}^{-i \theta_{k}} z}{1-r \mathrm{e}^{-i \theta_{k} z}}
$$

where $\theta_{k}=\frac{2 k \pi}{p}$, for $k=1,2, \ldots, p$.

a) Then, for every $z \in \mathbb{D}$ with $|z|=r$,

$$
|G(z)| \leq \frac{2 r^{p}}{1+r^{2 p}}=1-\frac{\left(1-r^{p}\right)^{2}}{1+r^{2 p}} .
$$


b) If besides we have $p h \leq 1 / 2$, where $h=1-r$, we also have, for every $z \in \mathbb{D}$ with $|z|=r$,

$$
|G(z)| \leq 1-\frac{(p h)^{2}}{2 \mathrm{e}} .
$$

Let us continue the proof of the theorem. Define $\chi:(0,1) \rightarrow(0,1]$ by

$$
\chi(x)=\sup _{t \leq x}[\max \{2 \delta(t), \sqrt{t}\}] .
$$

Then $\chi$ is non-decreasing, $\lim _{x \rightarrow 0} \chi(x)=0$ and $\lim _{x \rightarrow 1} \chi(x)=1$. We can find a decreasing sequence $\left(h_{n}\right)_{n \geq 0}$ of points $h_{n} \in(0,1)$, such that $\chi\left(h_{n}\right) \leq 2^{-n}$. This sequence converges to 0 ; in fact, $\sqrt{h_{n}} \leq \chi\left(h_{n}\right) \leq 2^{-n}$, by (3.8), and hence

$$
h_{n} \leq 2^{-2 n} .
$$

We now define, for every $n \in \mathbb{N}$, a positive integer $p_{n}$, by

$$
p_{n}=\min \left\{p \in \mathbb{N} ; \frac{p^{2} h_{n}^{2}}{2 \mathrm{e}}>2^{-n}\right\}
$$

We have $p_{n}>1$ because $h_{n}^{2} / 2 \mathrm{e}<h_{n}^{2} \leq 2^{-4 n}$. So, for every $n$, we have $4\left(p_{n}-1\right)^{2} \geq p_{n}^{2}$, and then

$$
4 \cdot 2^{-n} \geq \frac{4\left(p_{n}-1\right)^{2} h_{n}^{2}}{2 \mathrm{e}} \geq \frac{p_{n}^{2} h_{n}^{2}}{2 \mathrm{e}}
$$

This yields, for $n \geq 7$, that $\left(p_{n} h_{n}\right)^{2} \leq 8 \mathrm{e} 2^{-n} \leq 1 / 4$. Therefore $p_{n} h_{n} \leq 1 / 2$, and we can use the estimate in part (b) of Lemma 3.3.

Now, for $n \geq 7$, let $B_{n}$ be the finite Blaschke product defined by (3.4), where $r_{n}=1-h_{n}$. Using b) in Lemma 3.3, the Maximum Modulus Principle and the definition of $p_{n}$ in (3.10), we have

$$
\left|B_{n}(z)\right| \leq 1-\frac{p_{n}^{2} h_{n}^{2}}{2 \mathrm{e}}<1-2^{-n}, \quad \text { for }|z| \leq r_{n}
$$

Consider then the Blaschke product $D$ defined by

$$
D(z)=\prod_{n=7}^{\infty} B_{n}(z) .
$$

This product is convergent since, by (3.11), we have

$$
\sum p_{n}\left(1-r_{n}\right)=\sum p_{n} h_{n} \leq \sum \sqrt{8 \mathrm{e} 2^{-n}}<+\infty
$$

Finally, take $N \in \mathbb{N}$ big enough to have $r_{6}^{N}<1 / 2$, and define

$$
B(z)=z^{N} D(z) .
$$


Thus $B$ is a Blaschke product, and, if $|z| \leq r_{6}$, we have, since $\delta(t) \leq 1 / 2$ :

$$
|B(z)| \leq\left|z^{N}\right| \leq r_{6}^{N}<1 / 2 \leq 1-\delta(1-|z|) .
$$

If $1>|z|>r_{6}$, there exists $k \geq 7$ such that $r_{k} \geq|z|>r_{k-1}$. Therefore, thanks to $(3.12)$,

$$
|B(z)| \leq|D(z)| \leq\left|B_{k}(z)\right| \leq 1-2^{-k} .
$$

On the other hand $r_{k} \geq|z|>r_{k-1}$ implies $h_{k} \leq 1-|z|<h_{k-1}$, and so

$$
\delta(1-|z|) \leq \frac{1}{2} \chi(1-|z|) \leq \frac{1}{2} \chi\left(h_{k-1}\right) \leq 2^{-k} .
$$

Combining (3.16) and (3.17) we get $|B(z)| \leq 1-\delta(1-|z|)$, when $1>|z|>r_{6}$. From this and (3.15), Theorem 3.1 follows.

Proof of Lemma 3.3. It is obvious that, for all $a, z \in \mathbb{C}$,

$$
\prod_{k=1}^{p}\left(z-a \mathrm{e}^{i \theta_{k}}\right)=z^{p}-a^{p}
$$

Using this we have

$$
G(z)=\prod_{k=1}^{p} \frac{r-\mathrm{e}^{-i \theta_{k}} z}{1-r \mathrm{e}^{-i \theta_{k}} z}=\prod_{k=1}^{p} \frac{z-r \mathrm{e}^{i \theta_{k}}}{r z-\mathrm{e}^{i \theta_{k}}}=\frac{z^{p}-r^{p}}{(r z)^{p}-1} .
$$

Now, if $|z|=r$, we can write $z^{p}=r^{p} u$, for some $u$ with $|u|=1$. Then $|G(z)|=$ $|T(u)|$, where $T$ is the Möbius transformation

$$
T(u)=\frac{r^{p}(u-1)}{r^{2 p} u-1} .
$$

This transformation $T$ maps the unit circle $\partial \mathbb{D}$ onto a circumference $C$. As $T$ maps the extended real line $\mathbb{R}_{\infty}$ to itself, and $\partial \mathbb{D}$ is orthogonal to $\mathbb{R}_{\infty}$ at the intersection points 1 and $-1, C$ is the circumference orthogonal to $\mathbb{R}_{\infty}$ crossing through the points $T(1)=0$ and $T(-1)=\alpha$. It is easy to see that $|w| \leq|\alpha|$, for every $w \in C$; consequently,

$$
|G(z)| \leq \sup _{u \in \partial \mathbb{D}}|T(u)|=|T(-1)|=\frac{2 r^{p}}{1+r^{2 p}} .
$$

This finishes the proof of the statement (a).

To prove part (b), observe that, $1+r^{2 p} \leq 2$, and so, for $|z|=r$,

$$
|G(z)| \leq 1-\frac{\left(1-r^{p}\right)^{2}}{1+r^{2 p}} \leq 1-\frac{\left(1-r^{p}\right)^{2}}{2} .
$$

Remember that $r=1-h$, so $r \leq \mathrm{e}^{-h}$, and $r^{p} \leq \mathrm{e}^{-p h}$. Thus $1-r^{p} \geq 1-\mathrm{e}^{-p h}$. Now, if $x \in[0,1 / 2]$, we have, by the Mean Value Theorem,

$$
1-\mathrm{e}^{-x} \geq \frac{x}{\sqrt{\mathrm{e}}}
$$


Since $p h \leq 1 / 2$, we can apply this last estimate to (3.19) to get, as promised,

$$
|G(z)| \leq 1-\frac{\left(1-\mathrm{e}^{-p h}\right)^{2}}{2} \leq 1-\frac{p^{2} h^{2}}{2 \mathrm{e}}
$$

ending the proof of Lemma 3.3.

Remark. The key point in the proof of Theorem 3.1 is the inequality (3.6) in Lemma 3.3. This inequality may be viewed as a consequence of the strong triangle inequality (applied to $a=z^{p}, b=r^{p}$ and $c=0$ )

$$
d(a, b) \leq \frac{d(a, c)+d(c, b)}{1+d(a, c) d(c, b)}
$$

for the pseudo-hyperbolic distance $d(u, v)=\frac{|u-v|}{|1-\bar{u} v|}$ on $\mathbb{D}$. Let us recall a proof for the convenience of the reader: by conformal invariance, we may assume that $c=0$; then

$$
1-[d(a, b)]^{2}=\frac{\left(1-|a|^{2}\right)\left(1-|b|^{2}\right)}{|1-\bar{a} b|^{2}} \geq \frac{\left(1-|a|^{2}\right)\left(1-|b|^{2}\right)}{(1+|a||b|)^{2}}=1-[d(|a|,-|b|)]^{2},
$$

so that

$$
d(a, b) \leq d(|a|,-|b|)=\frac{|a|+|b|}{1+|a||b|},
$$

proving $(3.20)$, since $d(a, 0)=|a|$ and $d(0, b)=|b|$.

\section{A compact composition operator with a surjective symbol}

A well-known result of H. J. Schwartz (Theorem 2.8 of [17]) asserts that the composition operator $C_{\varphi}: H^{\infty} \rightarrow H^{\infty}$ is compact if and only if $\|\varphi\|_{\infty}<1$. In particular, the compactness of $C_{\varphi}: H^{\infty} \rightarrow H^{\infty}$ prevents the surjectivity of $\varphi$. It may therefore be expected that, the bigger $\Psi$, the more difficult it will be to obtain both the compactness of $C_{\varphi}: H^{\Psi} \rightarrow H^{\Psi}$ and the surjectivity of $\varphi$. The following theorem asserts that this is possible, and the case $H^{\infty}$ appears really as a singular case (corresponding to an "Orlicz function" which is discontinuous and can take the value infinity).

Theorem 4.1. For every Orlicz function $\Psi$, there exists a symbol $\varphi: \mathbb{D} \rightarrow \mathbb{D}$ which is 4-valent and surjective and such that $C_{\varphi}: H^{\Psi} \rightarrow H^{\Psi}$ is compact. Moreover, $\varphi$ can be taken so as $C_{\varphi}: H^{2} \rightarrow H^{2}$ is in all Schatten classes $S_{p}\left(H^{2}\right), p>0$.

In the case of $H^{2}\left(\Psi(x)=x^{2}\right)$, B. MacCluer and J. Shapiro gave an example (Example 3.12 in [14]) based on the Riemann mapping theorem and on the fact that, for a finitely valent symbol $\varphi$, we have the equivalence

$$
C_{\varphi}: H^{2} \rightarrow H^{2} \text { compact } \Longleftrightarrow \lim _{|z| \lesseqgtr 1} \frac{1-|\varphi(z)|}{1-|z|}=\infty .
$$


A specific example is as follows. Take

$$
R=\left\{z=x+i y \in \mathbb{C} ; x>0 \text { and } \frac{1}{x}<y<\frac{1}{x}+4 \pi\right\},
$$

let $g: \mathbb{D} \rightarrow R$ be a Riemann map and set $\varphi=\mathrm{e}^{-g}$. Then, $\varphi$ is 2 -valent, $\varphi(\mathbb{D})=\mathbb{D}^{*}$ (where $\mathbb{D}^{*}=\mathbb{D} \backslash\{0\}$ ), and the validity of (4.1) is tested through the use of the JuliaCarathéodory theorem (see [16] for details). To get a fully surjective mapping $\varphi_{1}$, just compose $\varphi$ with the square of a Blaschke factor:

$$
\varphi_{1}(z)=B \circ \varphi, \text { with } B(z)=\left(\frac{z-\alpha}{1-\bar{\alpha} z}\right)^{2}, \quad \alpha \in \mathbb{D}^{*}=\mathbb{D} \backslash\{0\}
$$

(note that $B(0)=B\left(2 \alpha / 1+|\alpha|^{2}\right)$. Since $C_{\varphi_{1}}=C_{\varphi} \circ C_{B}$, we see that $C_{\varphi_{1}}$ is compact as well and we are done.

Here, we can no longer rely on the Julia-Carathéodory theorem. But we shall use the following necessary and sufficient condition, in terms of the maximal Carleson function $\rho_{\varphi}$, which is valid for any symbol, finitely-valent or not (see Theorem 4.18 of [8] - or Théorème 4.2 of [7], where a different, but equivalent, formulation is given):

$$
C_{\varphi}: H^{\Psi} \rightarrow H^{\Psi} \text { compact } \Longleftrightarrow \lim _{h \rightrightarrows 0} \frac{\Psi^{-1}(1 / h)}{\Psi^{-1}\left(1 / \rho_{\varphi}(h)\right)}=0 .
$$

For the sequel, we shall set

$$
\Delta(h)=\frac{\Psi^{-1}(1 / h)}{\Psi^{-1}\left(1 / \rho_{\varphi}(h)\right)} .
$$

Our strategy will be to elaborate on the previous example to produce a (nearly) surjective $\varphi$ such that $\rho_{\varphi}(h)$ is very small (depending on $\Psi$ ) for small $h$. The tool will be the notion of harmonic measure for certain open sets of the extended plane $\hat{\mathbb{C}}=\mathbb{C} \cup\{\infty\}$, called hyperbolic (see Definition 19.9.3 in [2]); for example, every conformal image of $\mathbb{D}$ is hyperbolic (see Proposition 19.9.2 (d) and Theorem 19.9.7 of [2]). If $G$ is a hyperbolic domain and $a \in G$, the harmonic measure of $G$ at $a$ is the probability measure $\omega_{G}(a,$.$) supported by \partial G$ (here, and throughout the rest of this section, boundaries and closures will be taken in $\widehat{\mathbb{C}}$ ) such that

$$
u(a)=\int_{\partial G} u(z) d \omega_{G}(a, z)
$$

for each bounded and continuous function $u$ on $\bar{G}$, which is harmonic in $G$ (see Definition 21.1.3 in [2]). The harmonic measure at $a$ of a Borel set $A \subseteq \partial G$ will be denoted by $\omega_{G}(a, A)$. Clearly,

$$
\omega_{\mathbb{D}}(0, .)=m
$$

the Haar measure (i.e. normalized Lebesgue measure) of $\partial \mathbb{D}$. 
R. Nevanlinna (see Proposition 21.1.6 in [2]) showed that harmonic measures share a conformal invariance property. Namely, assume that $G$ is a simply connected domain, in which the Dirichlet problem can be solved (a Dirichlet domain), and $\tau: \overline{\mathbb{D}} \rightarrow \bar{G}$ is a continuous function which maps conformally $\mathbb{D}$ onto $G$; then $\tau$ maps $\partial \mathbb{D}$ onto $\partial G$, and, if $\tau(0)=a$ :

$$
\omega_{G}(a, A)=m\left(\tau^{-1}(A)\right)
$$

for every Borel set $A \subseteq \partial G$. This explains why harmonic measures enter the matter when we consider composition operators $C_{\varphi}$ : such an operator induces a map $H^{\Psi} \rightarrow L^{\Psi}\left(m_{\varphi}\right)$, where $m_{\varphi}=\varphi^{*}(m)$ appears as an image measure of $m$, as it happens for the harmonic measure of $G$ at $a$ in (4.5).

Lemma 4.2 (Hole principle). Let $G_{0}$ and $G_{1}$ be two hyperbolic open sets and $H \subseteq \partial G_{0}$ a Borel set such that

$$
G_{0} \subseteq G_{1} \quad \text { and } \quad \partial G_{0} \subseteq \partial G_{1} \cup H .
$$

Then, for every $a \in G_{0}$, we have the following inequality:

$$
\omega_{G_{1}}\left(a, \partial G_{1} \backslash \partial G_{0}\right) \leq \omega_{G_{0}}(a, H) .
$$

Proof. $^{2}$ By using Corollary 21.1.14 of [2], with $\Delta=\partial G_{0} \cap \partial G_{1}$, one has $\omega_{G_{0}}(a, \Delta) \leq$ $\omega_{G_{1}}(a, \Delta)$. But $\partial G_{1} \backslash \Delta=\partial G_{1} \backslash \partial G_{0}$, and hence, since harmonic measures are probability measures,

$$
\omega_{G_{1}}\left(a, \partial G_{1} \backslash \partial G_{0}\right)=\omega_{G_{1}}\left(a, \partial G_{1} \backslash \Delta\right)=1-\omega_{G_{1}}(a, \Delta) \leq 1-\omega_{G_{0}}(a, \Delta) ;
$$

we get the result since $\partial G_{0}=H \cup \Delta$, which implies $1 \leq \omega_{G_{0}}(a, H)+\omega_{G_{0}}(a, \Delta)$.

We also shall need the following result (see Proposition 21.1.17 in [2]).

Proposition 4.3 (Continuity principle). If $G$ is a hyperbolic open set and $a \in G$, then the harmonic measure $\omega_{G}(a,$.$) is atomless.$

Proof of Theorem 4.1. It will be enough to construct a 2-valent mapping $\varphi: \mathbb{D} \rightarrow \mathbb{D}$ such that $\varphi(\mathbb{D})=\mathbb{D}^{*}$ and $C_{\varphi}: H^{\Psi} \rightarrow H^{\Psi}$ is compact. We can then modify $\varphi$ by the same trick as the one used by B. MacCluer and J. Shapiro. Note that every point in $\mathbb{D}^{*}$ is the image by $\mathrm{e}^{-z}$ of two distinct points of $R$, except those which are the image of points of the hyperbola $y=(1 / x)+2 \pi$, which have only one pre-image.

For a positive integer $n$, set

$$
b_{n}=\frac{1}{4 n \pi},
$$

\footnotetext{
${ }^{2} \mathrm{~A}$ more intuitive way to see why this result holds is to use the fact, due to $\mathrm{S}$. Kakutani, and completed by J. Doob (see page 454 of [19] and Appendix F, page 477 of [6]) that the harmonic measure of $A$ at $a$ is the probability that the Brownian motion starting at $a$ exits from $G$ through the Borel set $A \subseteq \partial G$.
} 
and let $\varepsilon_{n}>0$ such that

$$
\frac{\Psi^{-1}\left(2 / b_{n+1}\right)}{\Psi^{-1}\left(1 / \varepsilon_{n}\right)} \leq \frac{1}{n} .
$$

We now modify the domain $R$, including "barriers" in it (not in the sense of potential theory, nor of Perron!) in the following way.

Let, for every $n \geq 1, M_{n}$ be the intersection point of the horizontal line $y=4 \pi n$ and of the hyperbola $y=(1 / x)+2 \pi$, that is $M_{n}=\frac{1}{4 \pi n-2 \pi}+4 \pi n i$.

Define inductively closed sets $P_{n}^{+}$and $P_{n}^{-}$, which are like small points of swords (two segments and a piece of hyperbola), in the following way:

- The lower part of $P_{n}^{+}$and $P_{n}^{-}$are horizontal segments of altitude $4 n \pi$.

- Those two horizontal segments are separated by a small open horizontal segment $H_{n}$ whose middle is $M_{n}$.

- The upper part of $P_{n}^{+}$is a slant segment whose upper extremity $c_{n}^{+}$lies on the hyperbola $y=1 / x$.

- The upper part of $P_{n}^{-}$is a slant segment whose upper extremity $c_{n}^{-}$lies on the hyperbola $y=(1 / x)+4 \pi$.

- The curvilinear part of $P_{n}^{+}$is supported by the hyperbola $y=1 / x$.

- The curvilinear part of $P_{n}^{-}$is supported by the hyperbola $y=(1 / x)+4 \pi$.

- One has $4(n+1) \pi-\mathfrak{I m} c_{n}^{ \pm}>2 \pi$.

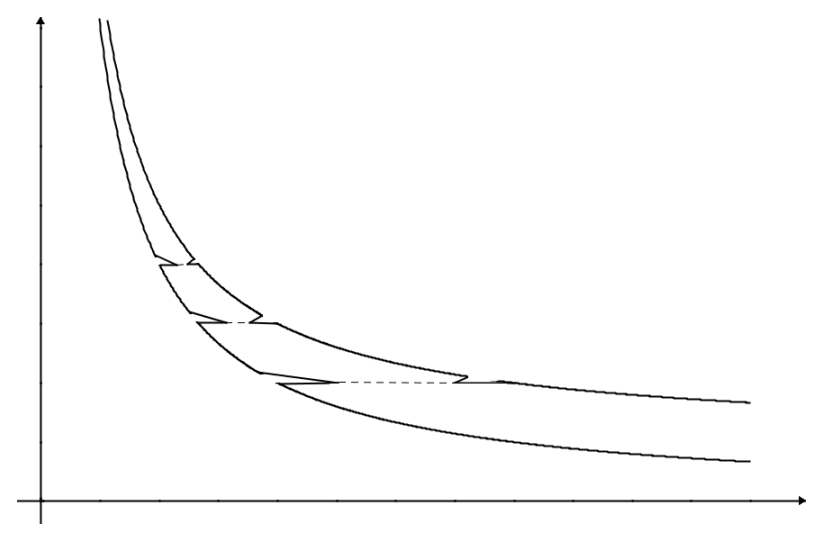

The size of the small horizontal holes will be determined inductively in the following way. Fix once and for all $a \in R$ such that $\operatorname{Im} a<4 \pi$. Suppose that $H_{1}, H_{2}, \ldots, H_{n-1}$ have already been determined. Set

$$
\Omega_{n}=\left\{z \in R \backslash \bigcup_{j<n}\left(P_{j}^{+} \cup P_{j}^{-}\right) ; \Im \mathrm{Im} z<4 n \pi\right\} .
$$

We can adjust $H_{n}$ so small that

$$
\omega_{\Omega_{n}}\left(a, H_{n}\right) \leq \varepsilon_{n}
$$


Indeed, $\Omega_{n}$ is bounded above by the horizontal segment $\left[b_{n}+4 i n \pi, b_{n-1}+4 i n \pi\right]$, where the point $M_{n}$ lies. If $H_{n}=\left[M_{n}-\delta, M_{n}+\delta\right]$, we see that $H_{n}$ decreases to the singleton $\left\{M_{n}\right\}$ as $\delta$ decreases to zero. Therefore, by Proposition 4.3, we can adjust $\delta$ so as to realize (4.10).

We now define our modified open set $\Omega$ by the formula

$$
\Omega=R \backslash \bigcup_{n \geq 1}\left(P_{n}^{+} \cup P_{n}^{-}\right)=\bigcup_{n \geq 1} \Omega_{n} .
$$

It is useful to observe that

$$
\inf _{w \in \partial \Omega_{n}} \text { Re } w=b_{n} .
$$

This is obvious by the way we defined the upper part of $\partial \Omega_{n}$.

Now, we can easily finish the proof. Fix $h \leq b_{1} / 2$ and let $n$ be the integer such that

$$
b_{n+1}<2 h \leq b_{n} .
$$

Let $g: \mathbb{D} \rightarrow \Omega$ be a conformal mapping such that $g(0)=a$. Since $\partial_{\infty} \Omega$ is connected, Caratheodory's Theorem (see [15]) ensures that $g$ can be continuously extended from $\overline{\mathbb{D}}$ onto $\bar{\Omega}$. More explicitly, using the Möbius transformation $T(z)=1 / z$, we see that there exists an automorphism of the extended complex plane such that $\bar{\Omega}$ is sent onto a compact subset of $\mathbb{C}$; so, we can apply to $\Omega$ many results stated for bounded domains. For instance, the boundary of $\Omega$ is a continuous path in the extended plane; so, by Theorem 14.5.5 of [2], $g$ can be extended to a continuous function (for the extended plane topology) $g: \overline{\mathbb{D}} \rightarrow \bar{G}$. In particular, $g$ has boundary values $g^{*}$.

We define $\varphi=\mathrm{e}^{-g}$.

As in the proof of B. MacCluer and J. Shapiro ([14]), we have that $\varphi$ is 2 -valent (see the remark made at the beginning of this proof), and we still have $\varphi(\mathbb{D})=\mathbb{D}^{*}$, since, in the process for constructing $\Omega$ from $R$, for every point of $\mathbb{D}^{*}$, at least one of the preimages by $\mathrm{e}^{-z}$ in $R$ has not been removed. Observe that, in particular, we did not remove any point in the hyperbola $y=(1 / x)+2 \pi$, thanks to the choice of $M_{n}$.

Moreover, $\Omega$ is a Dirichlet domain (because each component of $\partial \Omega$ has more than one point: see the comment after Definition 19.7.1 in [2]), so we can use the conformal invariance. Then by (4.5), (4.10), (4.12) and by the hole principle, we see that, if $A=\left\{\Re\right.$ e $\left.g^{*}\left(\mathrm{e}^{i t}\right)<2 h\right\}$,

$$
\begin{aligned}
\rho_{\varphi}(h) & \leq m_{\varphi}(\{|z|>1-h\})=m\left(\left\{\mathrm{e}^{-\Re e g^{*}\left(\mathrm{e}^{i t}\right)}>1-h\right\}\right) \\
& =m\left(\left\{\Re \operatorname{Re} g^{*}\left(\mathrm{e}^{i t}\right)<\log (1 / 1-h)\right\}\right) \\
& \leq m\left(\left\{\mathfrak{R e} g^{*}\left(\mathrm{e}^{i t}\right)<2 h\right\}\right)=\omega_{\mathbb{D}}(0, A) \\
& =\omega_{g(\mathbb{D})}(g(0), g(A))=\omega_{\Omega}(a,\{\mathfrak{R e} w<2 h\}) \\
& \leq \omega_{\Omega}\left(a,\left\{\mathfrak{R e} w \leq b_{n}\right\}\right) \\
& \leq \omega_{\Omega}\left(a, \partial \Omega \backslash \partial \Omega_{n}\right) \leq \omega_{\Omega_{n}}\left(a, H_{n}\right) \leq \varepsilon_{n} .
\end{aligned}
$$


It remains to observe that

$$
\Delta(h)=\frac{\Psi^{-1}(1 / h)}{\Psi^{-1}\left(1 / \rho_{\varphi}(h)\right)} \leq \frac{\Psi^{-1}\left(2 / b_{n+1}\right)}{\Psi^{-1}\left(1 / \varepsilon_{n}\right)} \leq \frac{1}{n} \leq C h,
$$

in view of (4.8) and of the choice of $n, C$ being a numerical constant. We should point out the fact that we applied the hole principle to the domains $G_{0}=\Omega_{n}$ and $G_{1}=\Omega$ and that this was licit because the assumptions of the hole principle (in particular the inclusion $\left.\partial \Omega_{n} \subseteq \partial \Omega \cup H_{n}\right)$ are satisfied. We have therefore proved that

$$
\lim _{h \rightrightarrows 0} \Delta(h)=0,
$$

and this ends, as we already explained, the first part of the proof of Theorem 4.1.

To prove the last part, let us remark that in (4.8) we may take $\varepsilon_{n}$ arbitrarily small. If one takes $\varepsilon_{n} \leq \mathrm{e}^{-n}$, one has, for some constant $c>0, \rho_{\varphi}(h) \leq \mathrm{e}^{-c / h}$, by using (4.13) and (4.14). In particular, $\rho_{\varphi}(h) \leq C h^{\alpha}$ for every $\alpha>1$. By Luecking's criterion, that implies that $C_{\varphi} \in S_{p}\left(H^{2}\right)$ for every $p>0$ (see Corollary 3.2 in [9]).

Remark. Let us note that our result is stronger than MacCluer-Shapiro's, since our $C_{\varphi}$ is in all Schatten classes $S_{p}\left(H^{2}\right), p>0$. Though our construction follows MacCluer-Shapiro's, it is the introduction of the "barriers" $P_{n}^{+}$and $P_{n}^{-}$which allows us to get this improvement.

\section{Composition operators with closed range}

In [1], J. Cima, J. Thomson and W. Wogen gave a characterization of composition operators $C_{\varphi}: H^{p} \rightarrow H^{p}$ with closed range. This characterization involves the Radon-Nikodym derivative of the restriction to $\partial \mathbb{D}$ of $m_{\varphi}$. They found it not satisfactory, and asked a characterization with the range of $\varphi$ itself. N. Zorboska ([20]) gave such a characterization, but her statement is somewhat complicated. We shall give here more explicit characterizations, either in terms of the Nevanlinna counting function $N_{\varphi}$, or in terms of the Carleson measure $m_{\varphi}$.

Theorem 5.1. Let $\varphi: \mathbb{D} \rightarrow \mathbb{D}$ be a non-constant analytic self map. Then the composition operator $C_{\varphi}: H^{p} \rightarrow H^{p}, 1 \leq p<\infty$, has closed range if and only if there is a constant $c>0$ such that, for $0<h<1$,

$$
\frac{1}{A(S(\xi, h))} \int_{S(\xi, h)} N_{\varphi}(z) d A(z) \geq c h, \quad \forall \xi \in \partial \mathbb{D} .
$$

We shall use the following result (Theorem 4.2 of [11]).

Theorem 5.2. There are universal constants $C, c>1$ such that

$$
m_{\varphi}(S(\xi, h)) \leq C \frac{1}{A(S(\xi, c h))} \int_{S(\xi, c h)} N_{\varphi}(z) d A(z)
$$

for every analytic self-map $\varphi: \mathbb{D} \rightarrow \mathbb{D}$, every $\xi \in \partial \mathbb{D}$, and $0<h<(1-|\varphi(0)|) / 8$. 
Then Theorem 5.1 will follow immediately from this theorem and the next theorem, applied to $\mu=m_{\varphi}$.

Theorem 5.3. Let $\mu$ be a finite positive measure on $\overline{\mathbb{D}}$. Assume that the canonical map $J: H^{p} \rightarrow L^{p}(\mu)$ is continuous, $1 \leq p<\infty$. Then $J$ is one-to-one and has a closed range if and only if there is a constant $c>0$ such that, for $0<h<1$,

$$
\mu[W(\xi, h)] \geq c h, \quad \forall \xi \in \partial \mathbb{D} .
$$

Proof. 1) Assume that $J$ has a closed range. By making a rotation on the variable $z$, we only have to find a constant $c>0$ such that

$$
\mu\left(S_{h}\right) \geq c h
$$

for $h>0$ small enough, where $S_{h}=S(1, h)$.

Since $J$ is one-to-one with closed range, the open mapping theorem implies that there is a constant $C>0$ such that

$$
\|f\|_{L^{p}(\mu)}^{p} \geq C^{p}\|f\|_{p}^{p}, \quad \forall f \in H^{p}
$$

We are going to test (5.4) on

$$
f_{N}(z)=\left(\frac{1+z}{2}\right)^{N}
$$

It is classical that there is a constant $c_{p}>0$ such that

$$
\left\|f_{N}\right\|_{p}^{p}=\int_{-\pi}^{\pi}\left|\cos \frac{t}{2}\right|^{p N} d t \geq \frac{c_{p}}{\sqrt{N}} .
$$

Now, since $|z+1|^{2}+|z-1|^{2}=2\left(|z|^{2}+1\right) \leq 4$ for every $z \in \overline{\mathbb{D}}$, one has

$$
\left|f_{N}(z)\right| \leq\left(1-\frac{|z-1|^{2}}{4}\right)^{N / 2} \leq \mathrm{e}^{-\frac{N}{8}|z-1|^{2}}
$$

Hence, using $\left|f_{N}(z)\right| \leq 1$ when $|z-1| \leq h$, one has

$$
\begin{aligned}
\left\|f_{N}\right\|_{L^{p}(\mu)}^{p} & \leq \mu\left(S_{h}\right)+\int_{|z-1|>h} \mathrm{e}^{-p \frac{N}{8}|z-1|^{2}} d \mu \\
& =\mu\left(S_{h}\right)+\int_{0}^{\mathrm{e}^{-p N h^{2} / 8}} \mu\left(\left\{\mathrm{e}^{-p \frac{N}{8}|z-1|^{2}}>u\right\}\right) d u,
\end{aligned}
$$

that is, making the change of variable $u=\mathrm{e}^{-p \frac{N}{8} x^{2}}$,

$$
\left\|f_{N}\right\|_{L^{p}(\mu)}^{p} \leq \mu\left(S_{h}\right)+\int_{h}^{\infty} \mu(\{|z-1| \leq x\}) \frac{p N}{4} x \mathrm{e}^{-p \frac{N}{8} x^{2}} d x .
$$


Now, the continuity of $J$ means, by Carleson's Theorem (see Theorem 9.3 in [4]), that there is a constant $K>0$ such that

$$
\sup _{|\xi|=1} \mu(S(\xi, x)) \leq K x, \quad 0 \leq x<1 \text {. }
$$

We get hence

$$
\begin{aligned}
\left\|f_{N}\right\|_{L^{p}(\mu)}^{p} & \leq \mu\left(S_{h}\right)+\int_{h}^{\infty} K x \frac{p N}{4} x \mathrm{e}^{-p \frac{N}{8} x^{2}} d x \\
& =\mu\left(S_{h}\right)+\frac{K \sqrt{8}}{\sqrt{p}} \frac{1}{\sqrt{N}} \int_{h \sqrt{\frac{p N}{8}}}^{\infty} y^{2} \mathrm{e}^{-y^{2}} d y .
\end{aligned}
$$

We take now for $N$ the smaller integer $>1 / h^{2}$, multiplied by some constant integer $a_{p}$, large enough to have

$$
\frac{K \sqrt{8}}{\sqrt{p}} \int_{\sqrt{\frac{p a_{p}}{8}}}^{\infty} y^{2} \mathrm{e}^{-y^{2}} d y \leq \frac{c_{p} C^{p}}{2} .
$$

We get then, from (5.4) and (5.6),

$$
\mu\left(S_{h}\right) \geq \frac{C^{p} c_{p}}{2} \frac{1}{\sqrt{N}},
$$

which gives (5.3).

2) Conversely, assume that (5.2) holds. Since the disk algebra $A(\mathbb{D})$ is dense in $H^{p}$, it suffices to show that there exists a constant $C>0$ such that $\|f\|_{L^{p}(\mu)} \geq$ $C\|f\|_{p}$ for every $f \in A(\mathbb{D})$.

Let $f \in A(\mathbb{D})$ such that $\|f\|_{p}=1$. Choose an integer $N$ such that

$$
\frac{1}{N} \sum_{n=1}^{N}\left|f\left(\mathrm{e}^{2 \pi i n / N}\right)\right|^{p} \geq \frac{1}{2} \int_{\partial \mathbb{D}}|f(\xi)|^{p} d m(\xi)=\frac{1}{2},
$$

and such that, due to the uniform continuity of $f$,

$$
z, z^{\prime} \in \overline{\mathbb{D}} \quad \text { and } \quad\left|z-z^{\prime}\right| \leq \frac{2 \pi}{N} \Longrightarrow\left|f(z)-f\left(z^{\prime}\right)\right| \leq \frac{1}{2^{(p+1) / p}} .
$$

Then, setting $W_{n}=W\left(\mathrm{e}^{2 \pi i n / N}, \pi / N\right), 1 \leq n \leq N$, one has

$$
\|f\|_{L^{p}(\mu)}^{p}=\int_{\overline{\mathbb{D}}}|f|^{p} d \mu \geq \sum_{n=1}^{N} \int_{W_{n}}|f|^{p} d \mu .
$$

If we choose $z_{n} \in W_{n}$ such that $\left|f\left(z_{n}\right)\right|=\min _{z \in W_{n}}|f(z)|$, we get, using (5.2),

$$
\|f\|_{L^{p}(\mu)}^{p} \geq \sum_{n=1}^{N}\left|f\left(z_{n}\right)\right|^{p} \mu\left(W_{n}\right) \geq \frac{c \pi}{N} \sum_{n=1}^{N}\left|f\left(z_{n}\right)\right|^{p} .
$$

Since $A^{p} \leq 2^{p-1}\left[(A-B)^{p}+B^{p}\right]$, by Hölder's inequality, one has

$$
\left|f\left(z_{n}\right)\right|^{p} \geq \frac{1}{2^{p-1}}\left|f\left(\mathrm{e}^{2 \pi i n / N}\right)\right|^{p}-\left|f\left(z_{n}\right)-f\left(\mathrm{e}^{2 \pi i n / N}\right)\right|^{p}
$$


and hence

$$
\|f\|_{L^{p}(\mu)}^{p} \geq \frac{c \pi}{N} \sum_{n=1}^{N}\left[\frac{1}{2^{p-1}}\left|f\left(\mathrm{e}^{2 \pi i n / N}\right)\right|^{p}-\left|f\left(z_{n}\right)-f\left(\mathrm{e}^{2 \pi i n / N}\right)\right|^{p}\right] .
$$

Now, since $z_{n} \in W_{n}$, one has

$$
\left|z_{n}-\mathrm{e}^{2 \pi i n / N}\right| \leq\left|z_{n}-\frac{z_{n}}{\left|z_{n}\right|}\right|+\left|\frac{z_{n}}{\left|z_{n}\right|}-\mathrm{e}^{2 \pi i n / N}\right| \leq \frac{\pi}{N}+\frac{\pi}{N}=\frac{2 \pi}{N}
$$

therefore $\left|f\left(z_{n}\right)-f\left(\mathrm{e}^{2 \pi i n / N}\right)\right| \leq 1 / 2^{p+1}$ and we get

$$
\begin{aligned}
\|f\|_{L^{p}(\mu)}^{p} & \geq c \pi\left[\frac{1}{N} \sum_{n=1}^{N} \frac{1}{2^{p-1}}\left|f\left(\mathrm{e}^{2 \pi i n / N}\right)\right|^{p}-\frac{1}{2^{p+1}}\right] \\
& \geq c \pi\left(\frac{1}{2^{p-1}} \frac{1}{2}-\frac{1}{2^{p+1}}\right)=\frac{c \pi}{2^{p+1}} .
\end{aligned}
$$

That ends the proof of Theorem 5.3.

Remark. Cima-Thomson-Wogen's criterion (Theorem 2 of [1]) says that if $\varphi$ is a non-constant analytic self-map of $\mathbb{D}$, the composition operator $C_{\varphi}$ has closed range if and only if the Radon-Nikodym derivative $d m_{\varphi} / d m$ is essentially bounded away from 0 . To make the link with this criterion, we shall see that condition (5.2) implies that the restriction of $\mu$ to the boundary $\mathbb{T}=\partial \mathbb{D}$ of the disk dominates the Lebesgue measure $m$. In fact, let $I$ be an $\operatorname{arc}$ of $\mathbb{T}$. If $m(I)=h$, we can write

$$
I=\bigcap_{n \geq 1} \bigcup_{j=1}^{n} W\left(\xi_{n, j}, h / 2 n\right)
$$

with disjoint windows $W\left(\xi_{n, 1}, h / 2 n\right), \ldots, W\left(\xi_{n, n}, h / 2 n\right)$; hence,

$$
\mu(I)=\lim _{n \rightarrow \infty} \sum_{j=1}^{n} \mu\left[W\left(\xi_{n, j}, h / 2 n\right)\right] \geq \lim _{n \rightarrow \infty} c \sum_{j=1}^{n} \frac{h}{2 n}=\frac{c}{2} h .
$$

\section{Composition operators in Schatten classes}

In [12], D. Luecking characterized composition operators $C_{\varphi}: H^{2} \rightarrow H^{2}$ which are in the Schatten classes, by using, essentially, the $m_{\varphi}$-measure of Carleson windows. Five years later, D. Luecking and K. Zhu ([13]) characterized them by using the Nevanlinna counting function of $\varphi$. We shall see in this section how the results of [11] (equivalence of the Carleson function of $\varphi$ with its Nevanlinna counting function: we shall be more explicit within the proof of Theorem 6.1) makes these two characterizations directly equivalent.

It will be convenient here to work with modified Carleson windows, namely

$$
W_{n, j}=\left\{z \in \overline{\mathbb{D}} ; 1-2^{-n} \leq|z| \leq 1 \text { and } \frac{(2 j-1) \pi}{2^{n}} \leq \arg z<\frac{(2 j+1) \pi}{2^{n}}\right\}
$$

$\left(j=0,1, \ldots, 2^{n}-1, n=1,2, \ldots\right)$. We shall say that $W_{n, j}$ is the Carleson window centered at $\mathrm{e}^{2 \pi i j / 2^{n}}$ with size $2^{-n}$. 
Theorem 6.1. For $p>0$ the two following conditions are equivalent:
a) $\frac{N_{\varphi}(z)}{\log (1 /|z|)} \in L^{p / 2}(\lambda)$, where $d \lambda(z)=(1-|z|)^{-2} d A(z)$ and $A$ is the norma- lized area measure on $\mathbb{D}$;
b) $\sum_{n=1}^{\infty} \sum_{j=0}^{2^{n}-1}\left[2^{n} m_{\varphi}\left(W_{n, j}\right)\right]^{p / 2}<\infty$.

Condition b) in the last theorem yields that $\lim _{n \rightarrow \infty} \max _{j} 2^{n} m_{\varphi}\left(W_{n, j}\right)=0$, and it is not difficult to see that this implies that $m_{\varphi}(\partial \mathbb{D})=0$, or equivalently, that $\left|\varphi^{*}\right|<1$ almost everywhere on $\partial \mathbb{D}$. In this situation we know (see Proposition 3.3 of [9]) that b) in Theorem 6.1 is equivalent to Luecking's condition in [12]. In fact the characterization of belonging to a Schatten class in [12] includes the requirement $m_{\varphi}(\partial \mathbb{D})=0$.

Proof. We may, and do, assume that $\varphi(0)=0$.

1) Assume first that condition b) is satisfied. Let

$$
R_{n, j}=\left\{z \in \mathbb{D} ; 1-\frac{1}{2^{n}} \leq|z|<1-\frac{1}{2^{n+1}} \text { and } \frac{(2 j-1) \pi}{2^{n}} \leq \arg z<\frac{(2 j+1) \pi}{2^{n}}\right\}
$$

be the (disjoint) Luecking windows $\left(0 \leq j \leq 2^{n}-1, n \geq 0\right)$. One has $R_{n, j} \subseteq W_{n, j}$.

By Theorem 3.1 of [11], there are a constant $C>0$ and an integer $K$ such that

$$
N_{\varphi}(z) \leq C m_{\varphi}\left(\widetilde{W}_{n, j}\right), \quad \text { for every } z \in R_{n, j}
$$

where $\widetilde{W}_{n, j}$ is the window centered at $\mathrm{e}^{2 \pi i j / 2^{n}}$, as $W_{n, j}$, but with size $2^{K-n}$. The windows $W_{n-K, j}, j=0,1, \ldots, 2^{n-K}-1$, have the same size as the windows $\widetilde{W}_{n, j}$, but may have a different center; nevertheless, each $\widetilde{W}_{n, j}$ can be covered with two windows $W_{n-K, l}$ : for $n>K$,

$$
\widetilde{W}_{n, j} \subseteq W_{n-K, l} \cup W_{n-K, l+1},
$$

for some $l=1,2, \ldots, 2^{n-K}$ (where $l+1$ is understood as 0 if $l=2^{n-K}-1$ ), we get (we shall use $\lesssim$ to mean $\leq$ up to a constant)

$$
\begin{aligned}
\int_{\mathbb{D}} \frac{\left(N_{\varphi}(z)\right)^{p / 2}}{(1-|z|)^{\frac{p}{2}+2}} d A(z) & \leq \sum_{n, j} \int_{R_{n, j}}\left(2^{n}\right)^{\frac{p}{2}+2}\left(N_{\varphi}(z)\right)^{p / 2} d A(z) \\
& \lesssim \sum_{n, j} \int_{R_{n, j}}\left(2^{n}\right)^{\frac{p}{2}+2}\left(m_{\varphi}\left(\widetilde{W}_{n, j}\right)\right)^{p / 2} d A(z) \\
& \lesssim \sum_{n, j}\left(2^{n}\right)^{p / 2}\left(m_{\varphi}\left(\widetilde{W}_{n, j}\right)\right)^{p / 2} \\
& \lesssim \sum_{\nu, l}\left(2^{\nu}\right)^{p / 2}\left(m_{\varphi}\left(W_{\nu, l}\right)\right)^{p / 2}<\infty
\end{aligned}
$$

and a) holds. 
2) Conversely, assume that a) is satisfied. We shall use the following inequality, whose proof will be postponed (for $p \geq 2$, (6.1) follows directly from Theorem 4.2 of [11], see Theorem 5.2 above, and Hölder's inequality):

$$
\left[m_{\varphi}\left(W_{n, j}\right)\right]^{p / 2} \lesssim \frac{1}{A\left(\widetilde{W}_{n, j}\right)} \int_{\widetilde{W}_{n, j}}\left[N_{\varphi}(z)\right]^{p / 2} d A(z)
$$

where $\widetilde{W}_{n, j}$ is a window with the same center as $W_{n, j}$ but with a bigger proportional size; say of size $2^{-n+L}$. We get

$$
\begin{aligned}
\sum_{n, j}\left[2^{n} m_{\varphi}\left(W_{n, j}\right)\right]^{p / 2} & \lesssim \sum_{n, j} 2^{n p / 2} 2^{2 n} \int_{\widetilde{W}_{n, j}}\left[N_{\varphi}(z)\right]^{p / 2} d A(z) \\
& =\int_{\mathbb{D}}\left(\sum_{n} 2^{n\left(2+\frac{p}{2}\right)}\left[\sum_{j} \mathbb{I}_{\widetilde{W}_{n, j}}(z)\right]\right)\left[N_{\varphi}(z)\right]^{p / 2} d A(z)
\end{aligned}
$$

Let $k=0,1, \ldots$ such that $1-2^{-k+1}<|z| \leq 1-2^{-k}$. One has $z \in \widetilde{W}_{n, j}$ only if $n \leq k+L$, and then, for each such $n, z$ is at most in $2^{L}$ windows $\widetilde{W}_{n, j}$. It follows that

$$
\sum_{n} 2^{n\left(2+\frac{p}{2}\right)} \sum_{j} \mathbb{I}_{\widetilde{W}_{n, j}}(z) \leq 2^{(k+L+1)\left(2+\frac{p}{2}\right)} \times 2^{L} .
$$

But $|z| \geq 1-2^{-k+1}$ implies $2^{(k+L+1)\left(2+\frac{p}{2}\right)} \leq C_{p} /(1-|z|)^{2+\frac{p}{2}} ;$ hence,

$$
\sum_{n, j}\left[2^{n} m_{\varphi}\left(W_{n, j}\right)\right]^{p / 2} \lesssim \int_{\mathbb{D}} \frac{\left[N_{\varphi}(z)\right]^{p / 2}}{(1-|z|)^{\frac{p}{2}+2}} d A(z)<\infty
$$

and b) holds.

It remains to show (6.1).

By Theorem 4.1 of [11], we can find a window $W$ with the same center as $W_{n, j}$, but with greater size $\operatorname{ch}\left(h=2^{-n}\right.$ is the size of the window $\left.W_{n, j}\right)$, such that

$$
m_{\varphi}\left(W_{n, j}\right) \lesssim \sup _{w \in W} N_{\varphi}(w)
$$

There is hence some $w_{0} \in W$ such that:

$$
m_{\varphi}\left(W_{n, j}\right) \lesssim N_{\varphi}\left(w_{0}\right)
$$

Take $R=\left|w_{0}\right|+c h$ (one has $R \geq 1$ since $w_{0} \in W$ and $W$ has size $c h$ ) and set $\varphi_{0}(z)=\varphi(z) / R$. One has $N_{\varphi_{0}}(z)=N_{\varphi}(R z)$ for $|z|<1 / R$ and $N_{\varphi_{0}}(z)=0$ if $|z| \geq 1 / R$.

Let now $u$ be the upper subharmonic regularization of $N_{\varphi_{0}}$ (see Lemma 1 and its proof on page 1140 in [13]): $u$ is a subharmonic function on $\mathbb{D} \backslash\{0\}$ such that $u \geq N_{\varphi_{0}}$ and $u=N_{\varphi_{0}}$ almost everywhere, with respect to $d A$. 
A result of C. Fefferman and E. M. Stein (Lemma 2 of [5]), generously attributed by them to Hardy and Littlewood, asserts that for any $q>0$, there exists a constant $C=C(q)$ such that

$$
[u(a)]^{q} \leq \frac{C}{A(D(a, r))} \int_{D(a, r)}[u(z)]^{q} d A(z)
$$

for every nonnegative subharmonic function $u$ on a domain $G$ and every disk $D(a, r) \subseteq G$ (see also Lemma 3 in [13]).

If $\Delta$ is the disk centered at $w_{0} / R$ and of radius $1-\left|w_{0}\right| / R$ (which is contained in $\mathbb{D} \backslash\{0\}$ since $R>\left|w_{0}\right|$ ), one has, by (6.2),

$$
\begin{aligned}
{\left[N_{\varphi}\left(w_{0}\right)\right]^{p / 2} } & =\left[N_{\varphi_{0}}\left(w_{0} / R\right)\right]^{p / 2} \leq\left[u\left(w_{0} / R\right)\right]^{p / 2} \\
& \leq \frac{C}{A(\Delta)} \int_{\Delta}[u(z)]^{p / 2} d A(z) \\
& =\frac{C}{A(\Delta)} \int_{\Delta}\left[N_{\varphi_{0}}(z)\right]^{p / 2} d A(z) \\
& =\frac{C}{A(\Delta)} \int_{\Delta \cap D(0,1 / R)}\left[N_{\varphi}(R z)\right]^{p / 2} d A(z) \\
& =\frac{C}{A(\tilde{\Delta})} \int_{\tilde{\Delta} \cap \mathbb{D}}\left[N_{\varphi}(w)\right]^{p / 2} d A(w),
\end{aligned}
$$

where

$$
\tilde{\Delta}=D\left(w_{0}, R-\left|w_{0}\right|\right)=D\left(w_{0}, c h\right) .
$$

Since the center $w_{0}$ of $\tilde{\Delta}$ is in $\mathbb{D}, \tilde{\Delta} \cap \mathbb{D}$ contains more than a quarter of $\tilde{\Delta}$ (at least for $c h \leq 1$ ), and hence

$$
A(\tilde{\Delta} \cap \mathbb{D}) \geq A(\tilde{\Delta}) / 4=c^{2} h^{2} / 4 \pi
$$

Now, let $\tilde{W}_{n, j}$ be the window with the same center as $W_{n, j}$ and of size $2 c h$. Since $2 c h \geq c h+\left(1-\left|w_{0}\right|\right), \tilde{W}_{n, j}$ contains $\tilde{\Delta} \cap \mathbb{D}$ and $A\left(\tilde{W}_{n, j}\right) \approx h^{2} \approx A(\tilde{\Delta})(\approx$ meaning that the ratio is between two absolute constants). We therefore get

$$
\left[N_{\varphi}\left(w_{0}\right)\right]^{p / 2} \lesssim \frac{1}{A\left(\tilde{W}_{n, j}\right)} \int_{\tilde{W}_{n, j}}\left[N_{\varphi}(w)\right]^{p / 2} d A(w)
$$

proving (6.1).

\section{References}

[1] Cima, J. A., Thomson, J. And Wogen, W.: On some properties of composition operators. Indiana Univ. Math. J. 24 (1974), no. 3, 215-220.

[2] Conway, J. B.: Functions of one complex variable II. Graduate Texts in Mathematics 159, Springer-Verlag, New York, 1995. 
[3] Cowen, C. C. and MacCluer, B. D.: Composition operators on spaces of analytic functions. Studies in Advanced Mathematics, CRC Press, Boca Raton, FL, 1995.

[4] Duren, P. L.: Theory of $H^{p}$ spaces. Second edition. Dover Publications, New York, 2000.

[5] Fefferman, C. and Stein, E. M.: $H^{p}$ spaces of several variables. Acta Math. 129 (1972), no. 3-4, 137-193.

[6] Garnett , J. B. And Marshall, D. E.: Harmonic measure. New Mathematical Monographs 2, Cambridge University Press, Cambridge, 2005.

[7] Lefèvre, P., Li, D., Queffélec, H. and Rodríguez-Piazza, L.: Opérateurs de composition sur les espaces de Hardy-Orlicz. C. R. Math. Acad. Sci. Paris 344 (2007), no. 1, 5-10.

[8] Lefièvre, P., Li, D., Queffélec, H. And Rodríguez-Piazza, L.: Composition operators on Hardy-Orlicz spaces. Memoirs Amer. Math. Soc. 207 (2010), no. 974.

[9] Lefèvre, P., Li, D., Queffélec, H. and Rodríguez-Piazza, L.: Some examples of compact composition operators on $H^{2}$. J. Funct. Anal. 255 (2008), no. 11, 3098-3124.

[10] Lefèvre, P., Li, D., Queffélec, H. And Rodríguez-Piazza, L.: Compact composition operators on $H^{2}$ and Hardy-Orlicz spaces. J. Math. Anal. Appl. 354 (2009), no. 1, 360-371.

[11] Lefèvre, P., Li, D., Queffélec, H. and Rodríguez-Piazza, L.: Nevanlinna counting function and Carleson function of analytic maps. Math. Ann. 351 (2011), no. $2,305-326$.

[12] Luecking, D. H.: Trace ideal criteria for Toeplitz operators. J. Funct. Anal. 73 (1987), no. 2, 345-368.

[13] Luecking, D. H. And Zhu, K.: Composition operators belonging to the Schatten ideals. Amer. J. Math. 114 (1992), no. 5, 1127-1145.

[14] MacCluer, B. And Shapiro, J.: Angular derivatives and compact composition operators on the Hardy and Bergman spaces. Canad. J. Math. 38 (1986), no. 4, 878-906.

[15] Pommerenke, C.: Boundary behaviour of conformal maps. Grundlehren der Mathematischen Wissenschaften 299, Springer-Verlag, Berlin, 1992.

[16] Queffélec, H.: Composition operators on Hardy-Orlicz and Bergman-Orlicz spaces of the disk. In Hot topics in operator theory, Proc. OT Conference 2008 in Timisoara, 159-171. Theta Ser. Adv. Math. 9, Theta, Bucharest, 2008.

[17] Schwartz, H. J.: Composition operators on $H^{p}$. Ph.D. Thesis, University of Toledo, 1969.

[18] Shapiro, J. H.: Composition operators and classical function theory. Universitext, Tracts in Mathematics, Springer-Verlag, New York, 1993.

[19] Stroock, D. W.: Probability theory, an analytic view. Cambridge University Press, Cambridge, 1994.

[20] Zorboska, N.: Composition operators with closed range. Trans. Amer. Math. Soc. 334 (1994), no. 2, 791-801. 
Received January 27, 2010; revised February 7, 2011.

Pascal Lefèvre: Université Lille Nord de France, UArtois, Laboratoire de Mathématiques de Lens EA 2462, Fédération CNRS Nord-Pas-de-Calais FR 2956, 62300 Lens, France.

E-mail: pascal.lefevre@euler.univ-artois.fr

DAniel Li: Université Lille Nord de France, UArtois, Laboratoire de Mathématiques de Lens EA 2462, Fédération CNRS Nord-Pas-de-Calais FR 2956, Faculté des Sciences Jean Perrin, Rue Jean Souvraz, S.P. 18, 62300 Lens, France.

E-mail: daniel.li@euler.univ-artois.fr

Hervé Queffélec: Université Lille Nord de France, USTL, Laboratoire Paul Painlevé U.M.R. CNRS 8524, 59655 Villeneuve D’Ascq Cedex, France.

E-mail: Herve.Queffelec@univ-lille1.fr

Luis Rodríguez-Piazza: Universidad de Sevilla, Facultad de Matemáticas, Departamento de Análisis Matemático, Apartado de Correos 1160, 41080 Sevilla, Spain.

E-mail: piazza@us.es

Part of this work was made during the fourth-named author's visit to the University of Lille 1 and the University of Artois (Lens) in June 2009. He was partially supported by a Spanish research project MTM2006-05622. 